\title{
Correction to "Investigating the Influence of Tracer Kinetics on Competition-Kinetic Association Binding Assays: Identifying the Optimal Conditions for Assessing the Kinetics of Low-Affinity Compounds"
}

\author{
In the above article [Sykes DA, Jain P, and Charlton SJ (2019) Mol Pharmacol 96(3): \\ 378-393; DOI: https://doi.org/10.1124/mol.119.116764], text citations to four references were \\ incorrectly listed in the Introduction during the proofing process.
}

The HTML and PDF versions of the article have been corrected.

The compositor regrets this error and any inconvenience it may have caused. 